\title{
The Effect of Hospital Service Quality on Patient's Trust
}

\author{
Ehsan Zarei ${ }^{1}$; Abbas Daneshkohan ${ }^{1}$; Roghayeh Khabiri ${ }^{2}$; Mohammad Arab ${ }^{3, *}$ \\ ${ }^{1}$ Department of Public Health, Shahid Beheshti University of Medical Sciences, Tehran, IR Iran \\ ${ }_{2}$ Department of Public Health, Shahid Beheshti University of Medical Sciences, Tehran, IR Iran \\ ${ }^{3}$ National Institute of Health Research, Tehran University of Medical Sciences, Tehran, IR Iran \\ ${ }^{*}$ Corresponding Author: Mohammad Arab, Department of Health Management and Economics, Tehran University of Medical Sciences, Tehran, IR Iran. Tel: +98-9124199720, Fax: +98- \\ 2122432036, E-mail: e.zarei@sbmu.ac.ir \\ Received: January 10, 2014; Revised:June 18, 2014; Accepted: August 10, 2014
}

Background: The trust is meant the belief of the patient to the practitioner or the hospital based on the concept that the care provider seeks the best for the patient and will provide the suitable care and treatment for him/her. One of the main determinants of patient's trust is the service quality.

Objectives: This study aimed to examine the effect of quality of services provided in private hospitals on the patient's trust.

Patients and Methods: In this descriptive cross-sectional study, 969 patients were selected using the consecutive method from eight private general hospitals of Tehran, Iran, in 2010. Data were collected through a questionnaire containing 20 items (14 items for quality, 6 items for trust)and its validity and reliability were confirmed. Data were analyzed using descriptive statistics and multivariate regression. Results: The mean score of patients' perception of trust was 3.80 and 4.01 for service quality. Approximately $38 \%$ of the variance in patient trust was explained by service quality dimensions. Quality of interaction and process $(\mathrm{P}<0.001)$ were the strongest factors in predicting patient's trust, but the quality of the environment had no significant effect on the patients' degree of trust.

Conclusions: The interaction quality and process quality were the key determinants of patient's trust in the private hospitals of Tehran. To enhance the patients' trust, quality improvement efforts should focus on service delivery aspects such as scheduling, timely and accurate doing of the service, and strengthening the interpersonal aspects of care and communication skills of doctors, nurses and staff.

Keywords:Health Care Quality; Patient Trust; Hospital-Patient Relation

\section{Background}

Trust is one of the most important determinants of creating and maintaining sustainable long-term relationships between the provider and the customer in the services sector $(1,2)$, so far, received very little attention in models used in the Health Sector for the Customers' Evaluation of health care services. In the health service, the trust is meant to be the belief of the patient to the practitioner or the hospital based on the concept that the care provider seeks the best for the patient and will provide the suitable care and treatment for him/her (3). The patient's trust consists of two main dimensions: honesty and benevolence. Honesty means the patient's belief in meeting of his/her needs by the hospital (4), in other words, belief in that the hospital will keep its commitments and has the capacity to fulfill its promises. Benevolence means that the patient believes that the hospital has deep concerns about the patients' health, interested in the improvement of the patients' health status and makes its utmost attempt in this regard (5). In other words, a good provider of services will not work only for his own benefits and will not take actions with adverse consequences for his customer (4). The confiding party typically is vulnerable to some degree in a mutual relationship because the relationship consequences are unknown and of course important to him.
This vulnerability is more important in health care services, domain where the customer normally has little skill and technical knowledge about the care results. The trust is an important and critical element in the health care relationship between the service provider and the customer $(3,6)$ and is related to access and better use of the health services (7). The trust is effective on the health status through continuity of care, adherence to the treatment instructions and the willingness to seek health care $(6,8)$ and results in increasing satisfaction and loyalty as well as a recommendation of the care provider to others $(7,9)$. Trust also can reduce patient's costs of information searching about the prices and alternative service providers and strengthen a closer mutual relationship between the patient and service provider. The higher the patient's trust level, these costs would be lower and the patient's commitment to the hospital stronger (5). The trust will be built when one party believes that the other party's actions will lead to positive outcomes for him/her. This suggests that the service quality may make this belief (10). Also, in the related literature the service quality has been referred to as one of the most important determinants of the patient's trust and creating a relatively strong relationship between the service provider and the customer (11-14). To build the

Copyright ( 2015, Iranian Red Crescent Medical Journal. This is an open-access article distributed under the terms of the Creative Commons Attribution-NonCommercial 4.0 International License (http://creativecommons.org/licenses/by-nc/4.0/) which permits copy and redistribute the material just in noncommercial usages, provided the original work is properly cited. 
sufficient trust with the service provider, the customers' perception of his/her service quality must be positive (15). The service quality means the fulfillment of the patient's expectations, and the ability a service provider in satisfying the patient's needs and expectations will result in the customer's positive perception towards the capabilities of the service provider. When the perception of service quality is positive and the performance of the provider results in meeting customer's expectations, the customers will trust more in the provider's capabilities and competencies $(9,14)$. In contrast, if the service provider's performance is accompanied by low quality and failure in satisfying the customer's expectations, the customer's trust in competencies and capabilities of the service provider will decrease (14). In fact, when a customer trusts in an organization, he/she trusts to the quality of its goods and services. Findings of a study in the outpatient setting of UK showed that the quality of the physician-patient relationship and the interpersonal aspects of the care have a significant impact on the patient's trust (16). Another study undertaken in the United States showed that the relationship quality and the physician's affective and informational support have a strong effect on the cancer patient's trust in their practitioner (10). Chang and Chen have declared that the service quality has a positive and powerful effect on patient trust and commitment (9).

\section{Objectives}

On the basis of the researchers' investigation, no study had been undertaken before the present study in this area of research in Iran's health service sector. In the studies undertaken outside Iran, the effect of the service quality on the patient's trust has been confirmed $(5,9,11)$, but even in these studies the relationship between the two constructs have been investigated generally. While on the basis of the researchers' point of views, "service quality" is a multi-dimensional construct (17) and each dimension's effect on the trust must be considered separately. The aim of this study was to evaluate the impact of service quality dimensions on patient's trust in Tehran private hospitals.

\section{Patients and Methods}

This descriptive and analytical study is part of a comprehensive study about patient's perceptions of services provided by private hospitals in Tehran, Iran, in 2010. A number of 969 patients were selected from the eight private general hospitals of Tehran based on proportional to size principle(Table 1). Patients from each hospital were selected consecutively and all the discharged patients were interviewed during the study period. Being at the age of at least 14, being at least one day (24 hours) hospitalized and willingness to participate in the study were the criteria for entering into the study. The questionnaire was given to the patients on the day of discharge. The aims of the study were explained to the patient and they were assured of confidentiality of their personal information. As for the illiterate patients, a trained interviewer helped in filling out of the questionnaire. The questionnaire consisted of three parts. The first part including 7 items related to the demographic-social specifications of the patient (age, gender, education level, insurance coverage, previous hospitalization in the same hospital, length of stay and the health status at discharge). In the second part, for the evaluation of service quality, the modified SERVPERF questionnaire has been used (18). This questionnaire included 14 items in the form of 3 dimensions: the environment quality with 4 items (Q1-Q4), the process quality with 6 items (Q5-Q10), and the interaction quality with 4 items (Q11-Q14). The third part also included 6 items (T1-T6) for the evaluation of the patient's trust to the hospital that was adapted from the Moliner study (5). To evaluate the reliability of the study instrument, the Cronbach's alpha coefficient was calculated and the coefficients 0.938 and 0.927 for the "perceived service quality" and the "trust" indicative of stability and reliability measurement tools, respectively. To evaluate the perception level of the service quality and trust, the Likert scale $(1=$ totally disagree, $5=$ totally agree) was used. The patient's perceived service quality and trust mean variable scores were obtained from the total item score divided by the number of items. The data were analyzed using descriptive statistics, t-test and ANOVA tests and multivariate analysis in SPSS17.0 software (SPSS Inc., Chicago, Illinois, USA). This study was approved by the Research Ethics Committee, Tehran University of Medical Sciences (code:130/1293).

\section{Results}

\subsection{Sample Profile of the Study}

Based on the study findings, 528 patients (54.5\%) were female and 441 (45.5\%) were male. The mean age of the patients was $48 \pm 16.9$. About $7 \%$ of the patients (64 people) were illiterate, $48 \%$ ( 469 people) had an academic degree and $91 \%$ ( 886 people) of the patients had insurance protection. The average length of stay (ALS) in the hospital was $4.5 \pm 4.4$ days. About $33 \%$ of the patients (316 people) had a previous hospitalization history in the current hospital and $27 \%$ (261 people) had used the current hospital outpatient service, previously. Moreover, $7.5 \%$ (72 people) of the patients described their health status at discharge from the hospital as "excellent", 55\% (530 people) as "good" and $37.5 \%$ (367 people) as "moderate" and "bad".

\begin{tabular}{lccc}
\hline \multicolumn{4}{l}{ Table 1. Characteristics of the Selected Hospitals } \\
\hline Name & Number of Beds & Type & Size Category \\
\hline Khatam & 400 & General & Large \\
Atyieh & 350 & General & Large \\
Kasra & 300 & General & Large \\
Mehr & 256 & General & Large \\
Bahman & 200 & General & Large \\
Madaen & 165 & General & Medium \\
Jam & 150 & General & Medium \\
Pastour & 80 & General & Medium \\
\hline
\end{tabular}




\subsection{Descriptive Findings}

The mean scores of the 14 items of the service quality varied from 3.34 (Q11: polite and friendly staff) to 4.38 (Q1: well-dressed and well-groomed staff). Among the three dimensions of service quality, the highest average score (4.19) belonged to the environmental quality and the lowest average score (3.74) related to the interaction quality (Table 2). The total mean score of the patients' perception of the service quality was $4.01 \pm 0.61$ from 5 . Moreover, the mean score of the six items of "trust" was 3.75 (T4: concern and commitment of hospital staff to solve problems related to the health of the patients) up to 3.83 (T6: hospital's commitment to meet the needs and satisfaction of patients). The total mean score of "trust" was $3.80 \pm 0.70$ from 5 (Table 2). A comparison of the trust scores based on the patient's demographic variables showed that the insurance coverage $(\mathrm{P}=0.016)$, the hospital size $(\mathrm{P}=$ 0.016), and the health status at discharge $(\mathrm{P}<0.001)$ have a significant impact over the patient's trust; however, the variables like the age, length of stay, gender, the education level and previous hospitalization history played no significant role in this regard. The trust score in patients with insurance coverage was higher than those without insurance protection. The patients of large hospitals (over 150 beds) got a higher trust score than those hospitalized in medium hospitals (below 150 beds). Furthermore, the patients who described their health status as "excellent" had the highest score and those who described their status as "bad" had the lowest trust score. With the increase in the literacy level, the patient's trust was also increased, but this increase was not statistically significant. To evaluate the relative importance of each dimension of the service quality in prediction of the patient's trust, the linear regression analysis was performed. The aim of this analysis was to quantify the relationship between the dependent (trust) and independent (service quality dimensions) variables. Based on the regression results, the $\mathrm{R}^{2}$ rate of this study model was 0.378 and therefore approximately $38 \%$ of the patient's trust variance was explained by the service quality dimensions (Table 3 ). The regression coefficients showed that the regression model was statistically significant and two independent variables (process quality and interaction quality) were positively effective in the patient's trust; however, the environment quality did not significantly affect the patient's trust. A one-unit increase in the interaction quality increased the patient trust for about 0.36, but the same value for the process quality would be around 0.27 . Results of this study showed that the interaction quality was the most powerful factor effective in the patient's trust.

\begin{tabular}{|c|c|}
\hline Item/Dimension & Mean \pm SD \\
\hline Environment Quality (EQ) & $4.19 \pm 0.59$ \\
\hline Q1.Well-dressed and well-groomed staff & $4.83 \pm 0.53$ \\
\hline Q2. Clean and comfortable environment of the hospital & $4.32 \pm 0.58$ \\
\hline Q3. Modern and state-of-the-art equipment & $3.97 \pm 0.93$ \\
\hline Q4.Visual appeal of physical facilities & $4.14 \pm 0.75$ \\
\hline Process Quality (PQ) & $4.07 \pm 0.72$ \\
\hline Q5. Telling when services will be performed & $4.04 \pm 0.79$ \\
\hline Q6. Prompt provision of medical and non-medical services & $4.05 \pm 0.80$ \\
\hline Q7. Willingness of staff to help patients & $4.10 \pm 0.79$ \\
\hline Q8. The availability of staff when needed & $4.11 \pm 0.82$ \\
\hline Q9. Creating a sense of trust in the patient & $4.06 \pm 0.85$ \\
\hline Q10. Conducting the services right at the first time & $4.06 \pm 0.84$ \\
\hline Interaction Quality (IQ) & $3.74 \pm 0.79$ \\
\hline Q11. Polite and friendly dealing with patients by staff & $3.34 \pm 1.19$ \\
\hline Q12. Attention to the patients' beliefs and emotions & $3.91 \pm 0.82$ \\
\hline Q13. Having patients' best interest at heart & $3.85 \pm 0.85$ \\
\hline Q14. Understanding the specific needs of patients & $3.87 \pm 0.85$ \\
\hline Trust & $3.80 \pm 0.70$ \\
\hline T1. True claims about this hospital and its services & $3.80 \pm 0.72$ \\
\hline T2. Fulfillment of promised commitments by hospital & $3.79 \pm 0.74$ \\
\hline T3. Honest and trustworthy staff & $3.79 \pm 0.80$ \\
\hline T4. Concern and commitment of hospital staff to solve patient's problems & $3.75 \pm 0.80$ \\
\hline T5. Staff want the best for the patients & $3.81 \pm 0.79$ \\
\hline T6. Hospital's commitment to meet the needs and satisfaction of patients & $3.83 \pm 0.79$ \\
\hline
\end{tabular}


Zarei E et al.

\begin{tabular}{lcccc}
\hline \multicolumn{5}{l}{ Table 3. The Impact of Service Quality on Patient Trust } \\
\hline Quality Dimensions & B & Beta & t-value & Significance \\
\hline Constant coefficient & 1.25 & - & 9.663 & $<0.001$ \\
EQ & 0.07 & 0.06 & 1.657 & 0.098 \\
PQ & 0.26 & 0.27 & 8.838 & $<0.001$ \\
IQ & 0.32 & 0.36 & 10.601 & $<0.001$
\end{tabular}

a Abbreviations: B, Unstandardised Beta; EQ, Environment Quality; PQ,

Process Quality; IQ, Interaction Quality.

b Adjusted $\mathrm{R}^{2}=0.378 ; \mathrm{F}=195.09 \mathrm{P}<0.001$.

\section{Discussion}

The main objective of this study was to investigate the impact of service quality on the patient's trust in private hospitals. The average score of patient trust was 3.79 of 5; showing the great potential to improve patient's trust in the private hospitals of Tehran. The $\mathrm{R}^{2}$ value in this model was 0.38 and hence our model has a relatively good predictive power. Based on the Cohen recommendation, $\mathrm{R}^{2}$ values higher than 0.25 , indicating a large variance in the model (19). Considering the above results, it can be inferred that the hypothetical model has been effective in explaining the relationship between the "service quality" and "patient's trust" in private hospitals. The patients without insurance had lower trust score. In the previous studies, it was confirmed that the evaluation of the patients with insurance coverage compared with the patients without such protection is more positive concerning the services they receive from the hospital (20). The patient's health status was also influential on the patient's trust to the hospital and the patients who had evaluated their health status as good at discharge time, had a high-trust score. In the previous studies, it was also proved that patient's better physical and mental health status has a significant effect on their evaluation of the received services $(21,22)$. It seems if the patients feel more improvement in their health status at discharge time, they may have greater confidence in the hospital's ability in fulfilling their needs. The size of the hospital was another effective factor in the patient's trust level. Probably the patients imagine that the larger hospital means more facilities and resources, and consequently greater ability to meet the needs and expectations of the patients. Our findings showed that the process quality and the interaction quality had a direct and positive impact on the patient's trust, which is consistent with the results of the previous studies. Caceres and Paparoidamis's findings indicate that increasing the customers' perception about the service quality can affect some aspects of the relationship quality such as "trust" (12). Results of a study in Jordan's private hospitals showed that service quality has a positive and direct effect on the patient's trust. From among the service quality dimensions, "empathy" and "responsiveness" dimensions had the highest effect on the "trust" (11). Results of Moliner's study in Spain showed that hospital's service quality is the most important determinant of the patient's trust (5). Chang et al. in their study on Taiwan's hospitals showed that "service quality" perception has a strong positive impact on the patient's trust (9). Interaction between the customer and service provider constitutes the main core of the servicefocused businesses. The relatively strong effect of interaction quality on the patient's trust is in turn an indicative of the importance of the personnel and the physician's role in relationship with the patient, so that the more strong their interaction with the patient is, the more the patient's trust will be strengthened (7). The patients who have anxiety, fear and stress need the physicians and nurses to assign some of their time to speak to them and ease their concerns; give them the necessary information concerning the illness and the treatment options and assure their patients that they will do their utmost struggle to improve the patients' health status and provide them with the best care possible. In fact, an effort to create a friendly and warm relationship with the patients, listening to their concerns and attempting to resolve it as well as trying to meet their needs and expectations would be the more effective factors on patient's trust. Tarrant et al. in a study in the UK clinics claimed that the quality of the relationship between patient-physician and interpersonal aspects of care strongly has a strong effect on patient's trust (16). The ability of the hospital and its staff in providing the patient with quality, timely and accurate care will be strength the patient's belief in the ability and honesty of the service provider as well as positive perception of care quality and ultimately building trust in him. In fact the ability to provide the services in an accurate, correct and fast manner is an indication of the hospitals' ability to meet the patient's needs. The care providers therefore should be focused on the patient's health and convenience to establish a quality relationship. The physical environment's quality of the hospital had a small impact on the patient's trust, which is consistent with the results of other studies (11). This means that the presence of a clean environment and suitable hoteling and space in the hospital cannot attract the patient's trust and in fact hospital must show its ability to meet the patient's needs practically in terms of the manner of service delivery and the personnel-patient interaction. Our study findings showed that the patient's experience of the services provided by the private hospitalshas a strong impact on the outcome variables such as patient's trust. High-quality services significantly strengthen the "trust", which is effective on the patients' satisfaction and loyalty. "Process Quality" and "Interaction Quality" are key determinants of the patient's trust in Tehran private hospitals. If the private hospitals aim to enhance the patients' trust, the quality improvement efforts must focus on the managerial aspects of service delivery, such as scheduling, timely and careful doing of the services, and strengthening the interpersonal relationships as well as the communication skills of the physicians, nurses and other personnel. 
Our study has some limitations. First, since in this study the patients have evaluated the quality of services and their trust in the hospital at discharge time from the hospital, their judgments may have been affected by the degree of improvement of their health status and create a bias. So, it is recommended that interviewing with the patients as regards to their evaluation of different aspects of the hospital services is made at least one week after their discharge from the hospital so that more accurate results could be obtained. Second, evaluation of the quality and trust by the patient is subjective and using the questionnaire as a quantitative tool cannot reflect all the patient's judgments. Using qualitative methods therefore besides the quantitative methods in the future studies could provide better understanding of the evaluation of hospital services.

\section{Acknowledgements}

This study was a part of a PhD thesis supported financially by Tehran University of Medical Sciences; according to contract number 10477 dated May 23, 2010. We thank the individuals and organizations who participated in our research, especially managers, and other personnel of Atyieh, Kasra, Khatam, Madaen, Pastour, Mehr, Jam and Bahman Hospitals.

\section{Authors' Contributions}

Ehsan Zarei selected the topic and designed the study, analyzed the data, interpreted the findings, wrote the first draft of the manuscript and revised the manuscript; Abbas Daneshkohan analyzed the data, interpreted the findings and commented on the first draft of the manuscript; Roghayeh Khabiri analyzed the data, interpreted the findings and commented on the first draft of the manuscript; Mohammad Arab designed and supervised the study, analyzed the data, interpreted the findings, commented on the first draft of the manuscript and revised the manuscript.

\section{Funding/Support}

This study was a part of a PhD thesis supported financially by Tehran University of Medical Sciences, according to contract number 10477 dated May 23, 2010.

\section{References}

1. Deng Z, Lu Y, Wei KK, Zhang J. Understanding customer satisfaction and loyalty: An empirical study of mobile instant messages in China. Int J Inf Manag. 2010;30(4):289-300.

2. Akbar MM, Parvez N. Impact of service quality, trust, and customer satisfaction on customer loyalty. ABAC J. 2009;29(1):24-38.

3. Platonova EA, Kennedy KN, Shewchuk RM. Understanding patient satisfaction, trust, and loyalty to primary care physicians. Med Care Res Rev. 2008;65(6):696-712.

4. Naoui FB, Zaiem I. The impact of relationship quality on client's loyalty: An application in the parapharmaceutical industry. Int $J$ Pharm Healthc Marketing. 2010;4(2):137-56.

5. Moliner MA. Loyalty, perceived value and relationship quality in healthcare services. J Serv Manag. 2009;20(1):76-97.

6. Dugan E, Trachtenberg F, Hall MA. Development of abbreviated measures to assess patient trust in a physician, a health insurer, and the medical profession. BMC Health Serv Res. 2005;5:64.

7. Ozawa S, Sripad P. How do you measure trust in the health system? A systematic review of the literature. Soc Sci Med.2013;91:104.

8. Trachtenberg F, Dugan E, Hall MA. How patients' trust relates to their involvement in medical care. J Fam Pract. 2005;54(4):34452.

9. Chang CS, Chen SY, Lan YT. Service quality, trust, and patient satisfaction in interpersonal-based medical service encounters. BMC Health Serv Res. 2013;13:22.

10. Arora NK, Gustafson DH. Perceived helpfulness of physicians' communication behavior and breast cancer patients' level of trust over time. J Gen Intern Med. 2009;24(2):252-5.

11. Alrubaiee L, Alkaa'ida F. The Mediating Effect of Patient Satisfaction in the Patients' Perceptions of Healthcare Quality-Patient Trust Relationship. Int J Marketing Stud. 2011;3(1):103-27.

12. Caceres RC, Paparoidamis NG. Service quality, relationship satisfaction, trust, commitment and business-to-business loyalty. Eur J Marketing. 2007;41(7):836-67.

13. Liu CT, Guo YM, Lee $\mathrm{CH}$. The effects of relationship quality and switching barriers on customer loyalty. Int J Inf Manag. 2011;31(1):71-9.

14. Singh J, Sirdeshmukh D. Agency and trust mechanisms in consumer satisfaction and loyalty judgments. J Acad Marketing Sci. 2000;28(1):150-67.

15. Roostika R. The Effect of Perceived Service Quality and Trust on Loyalty: Customer's Perspectives on Mobile Internet Adoption. Int J Innov Manag Technol. 2011;2(4):286-91.

16. Tarrant C, Stokes T, Baker R. Factors associated with patients' trust in their general practitioner: a cross-sectional survey. $\mathrm{Br} J$ Gen Pract. 2003;53(495):798-800.

17. Jen W, Tu R, Lu T. Managing passenger behavioral intention: an integrated framework for service quality, satisfaction, perceived value, and switching barriers. Transp. 2011;38(2):321-42.

18. Cronin Jr JJ, Taylor SA. SERVPERF versus SERVQUAL: reconciling performance-based and perceptions-minus-expectations measurement of service quality. J Marketing.1994:125-31.

19. Cohen J. Statistical power analysis for the behavioral sciences.New Jersey: Lawrence Erlbaum; 1988.

20. Bakar C, Akgun HS, Al Assaf AF. The role of expectations in patients' hospital assessments: a Turkish university hospital example. Int J Health Care Qual Assur. 2008;21(5):503-16.

21. Da Costa D, Clarke AE, Dobkin PL, Senecal JL, Fortin PR, Danoff DS, et al. The relationship between health status, social support and satisfaction with medical care among patients with systemic lupus erythematosus. Int J Qual Health Care.1999;11(3):201-7.

22. Rahmqvist M. Patient satisfaction in relation to age, health status and other background factors: a model for comparisons of care units. Int J Qual Health Care. 2001;13(5):385-90. 